\section{A microcomputer program for analyzing multitrait-multimethod matrices}

\author{
TOSHI HAYASHI and RON D. HAYS \\ RAND Corporation, Santa Monica, California
}

A multitrait-multimethod (MTMM) matrix comprises intercorrelations of two or more traits measured by two or more methods. An examination of an MTMM matrix allows a thorough assessment of convergent and discriminant validity (Campbell \& Fiske, 1959). Unfortunately, this process involves an overwhelming number of comparisons of correlations. To minimize the difficulty in using the MTMM technique, we have written a FORTRAN program for the IBM and IBM-compatible microcomputers.

This paper presents a brief demonstration of our MTMM FORTRAN program, MTMM.EXE, to show the efficiency and usefulness of computerized analysis of MTMM matrices.

MTMM.EXE. An MTMM program originally was written in BASIC by Hays (1986). However, the program was intolerably slow. To improve upon execution time, as well as ease of use, the program was translated into FORTRAN. In the process, we considerably enhanced the program algorithm and output. MTMM.EXE provides a more thorough output, is easier to use, and runs considerably faster than the original BASIC program. The version described here, MTMM.EXE 2.2, is 56K in size and requires about $120 \mathrm{~K}$ of free memory (RAM) to run. This version can handle up to 15 traits and 10 methods. ${ }^{\text {' }}$ MTMM.EXE 2.2 runs under Microsoft Disk Operating System (MS-DOS) Version 2.0 or later on IBM PC or compatible (e.g., Compaq) microcomputers.

How to Use MTMM.EXE. To use MTMM.EXE, the user needs to create and save an ASCII file that contains two header lines, trait labels, and a MTMM matrix. Each header, label, and matrix component must start on a new line, and these items cannot be separated by blank lines.

The header portion of input consists of one line of title (maximum of 70 characters) and another line for the number of cases on which the correlation matrix is based, number of traits, number of methods, and number of digits for each correlation entry. Entries within a line are delimited by one or more blanks. The two header lines

Preparation of this article was supported in part by grants for the $\mathrm{Na}$ tional Study of Medical Care Outcomes from the Robert Wood Johnson Foundation, The Henry J. Kaiser Family Foundation, and the Pew Memorial Trust. The opinions expressed are those of the authors and do not necessarily reflect the views of the sponsors or the RAND Corporation.

Requests for reprints may be sent to Toshi Hayashi, RAND Corporation, 1700 Main St., Santa Monica, CA 90406-2138. can be optionally separated from one another by one or more blank lines.

The number of trait labels must coincide with the number of traits specified in the second header line. Each trait label must appear on a new line. Labels can be 70 characters long, but only the first 8 characters are used in labeling the output of the MTMM matrix.

The input MTMM matrix can be either a full square or a lower half matrix. Correlations should be delimited by one or more blanks. If the first line contains only one correlation entry, the program assumes that the input matrix is a lower half matrix. Otherwise, the input matrix is assumed to be a full square matrix. Each row of the correlation matrix, representing correlations involving a particular trait, must start on a new line.

An illustrative input file for MTMM.EXE is presented in Table 1 . The third line, following an optionally inserted blank line, informs that the number of cases is 286 , the number of traits is 5 , the number of methods is 3 , and the number of digits for correlation entries is 3 . The label part has five labels. This number corresponds to the number of traits given in the header. The first line of the MTMM matrix has only one correlation entry, indicating that the input matrix is a lower half matrix.

To run MTMM.EXE, type d:MTMM at the DOS prompt, where "d:" represents a drive specifier, necessary to specify if the program does not reside on the default drive. The user is prompted for several pieces of information, in the following order:

1. Input file name. The user provides a file name that has the input matrix and required information, as is done in the example presented in Table 1. The file name can be up to 15 characters long, including its drive specifier and path.

2. Output file name. The user provides the file name in which the results will be stored. The file name is up to 15 characters long.

3. Output style. One of three output styles is selected. The three styles differ in the volume of output generated. Namely, the user can select to generate (a) only the summary table of results, (b) all validity comparisons in addition to (a), or (c) a labeled output of the input matrix in addition to (b). This flexibility of output is one of the enhanced features of the latest version of the program.

4. Line width of input matrix printing. If output style $3 \mathrm{c}$ is selected, then the user can choose the line width of input matrix printing. The range is between 64 and 240 columns. The default size is 70 columns wide.

5 . Decimal points assignment. Finally, the user indicates whether the correlation entries in the input matrix already have decimal points or the program needs to assign decimal points.

Sample Run. Table 2 presents a portion of a sample output using the input file shown in Table 1, the MTMM 
Table 1

Illustrative MTMM.EXE Input

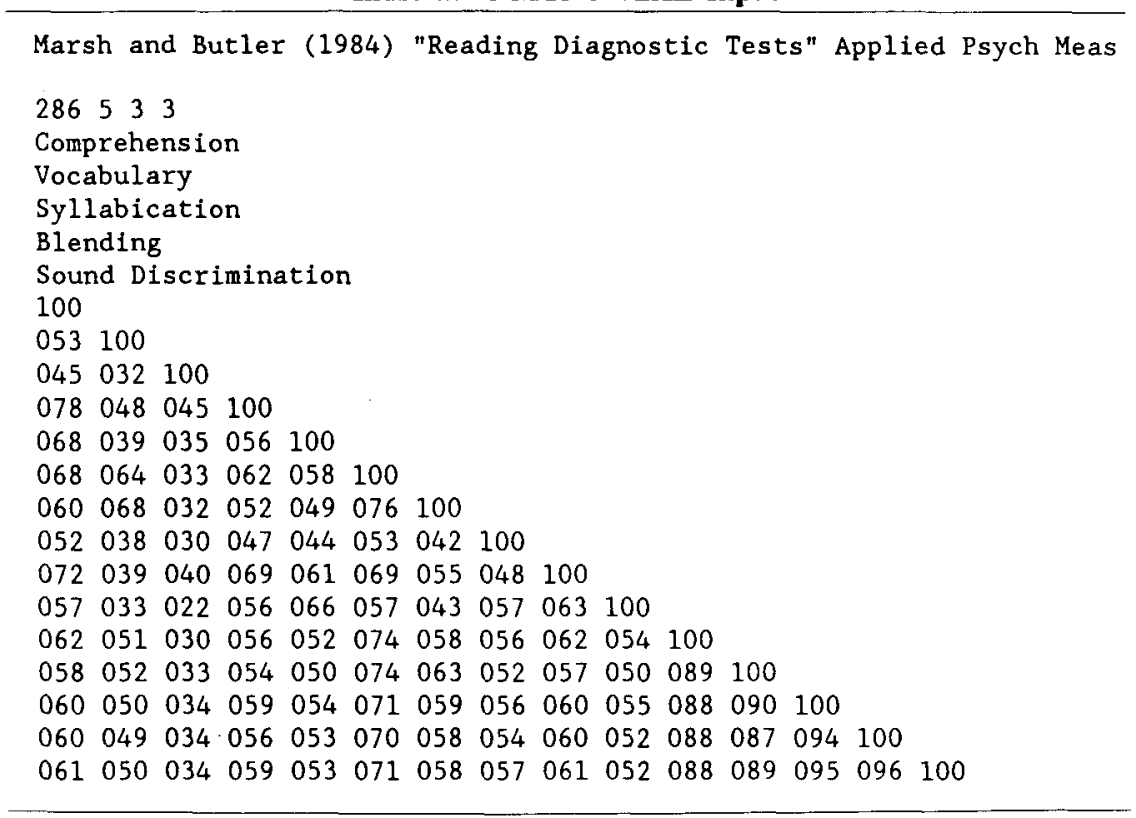

Table 2

A Portion of the MTMM.EXE Output

\begin{tabular}{|c|c|c|c|c|c|c|c|c|c|c|}
\hline \multicolumn{11}{|c|}{$\begin{array}{l}\text { MTMM.EXE (2.2): Multitrait-Multimethod Pr } \\
\text { Programmers: Ron Hays \& Toshi Hayashi, Ra }\end{array}$} \\
\hline \multicolumn{11}{|c|}{ Correlation Matrix Input Is As Follows: } \\
\hline Marsh & and Butler & c) $(1984$ & ) "Rea & ading $D$ & Diagnos & stic $\mathrm{Te}$ & ests" A & Applied & Psych & Meas \\
\hline \multirow[t]{3}{*}{$N=$} & $286 ; \mathrm{DFS}=$ & 283 & & & & & & & & \\
\hline & METHOD & 1 & & & & & 2 & & & \\
\hline & TRAIT & 1 & 2 & 3 & 4 & 5 & 1 & 2 & 3 & 4 \\
\hline \multicolumn{11}{|c|}{ 1. 1.Comprehe 1.00} \\
\hline \multicolumn{2}{|c|}{ 2.Vocabula } & .53 & 1.00 & & & & & & & \\
\hline & .45 & .32 & 1.00 & & & & & & \\
\hline \multicolumn{2}{|c|}{ 4. Blending } & .78 & .48 & .45 & 1.00 & & & & & \\
\hline \multirow{2}{*}{\multicolumn{2}{|c|}{$\begin{array}{l}\text { 5. Sound } \mathrm{Di} \\
\text { 2. } 1 \text {. Comprehe }\end{array}$}} & .68 & .39 & .35 & .56 & 1.00 & & & & \\
\hline & & {$[.68]$} & .64 & .33 & .62 & .58 & 1.00 & & & \\
\hline & Vocabula & .60 & {$[.68]$} & .32 & .52 & .49 & .76 & 1.00 & & \\
\hline & Syllabic & .52 & .38 & {$[.30]$} & .47 & .44 & .53 & .42 & 1.00 & \\
\hline & Blending & .72 & .39 & .40 & {$[.69]$} & .61 & .69 & .55 & .48 & 1.00 \\
\hline \multicolumn{2}{|c|}{ 5. Sound Di } & .57 & .33 & .22 & .56 & {$[.66]$} & .57 & .43 & .57 & .63 \\
\hline \multirow{7}{*}{3.} & Comprehe & {$[.62]$} & .51 & .30 & .56 & .52 & {$[.74]$} & .58 & .56 & .62 \\
\hline & Vocabula & .58 & {$[.52]$} & .33 & .54 & .50 & .74 & {$[.63]$} & .52 & .57 \\
\hline & Syllabic & .60 & .50 & {$[.34]$} & .59 & .54 & .71 & .59 & {$[.56]$} & .60 \\
\hline & Blending & .60 & .49 & .34 & {$[.56]$} & .53 & .70 & .58 & .54 & {$[.60]$} \\
\hline & Sound Di & .61 & .50 & .34 & .59 & {$[.53]$} & .71 & .58 & .57 & .61 \\
\hline & METHOD & 2 & 3 & & & & & & & \\
\hline & TRAIT & 5 & 1 & 2 & 3 & 4 & 5 & & & \\
\hline \multicolumn{3}{|c|}{ מ } & \multicolumn{8}{|c|}{ 2. 5.Sound Di 1.00} \\
\hline \multirow{5}{*}{$\begin{array}{r}1 \\
2 \\
3 \\
4 \\
5\end{array}$} & Comprehe & .54 & 1.00 & & & & & & & \\
\hline & Vocabula & .50 & .89 & 1.00 & & & & & & \\
\hline & Syllabic & .55 & .88 & .90 & 1.00 & & & & & \\
\hline & Blending & .52 & .88 & .87 & .94 & 1.00 & & & & \\
\hline & Sound Di & {$[.52]$} & .88 & .89 & .95 & .96 & 1.00 & & & \\
\hline
\end{tabular}


Table 2 (Continued)

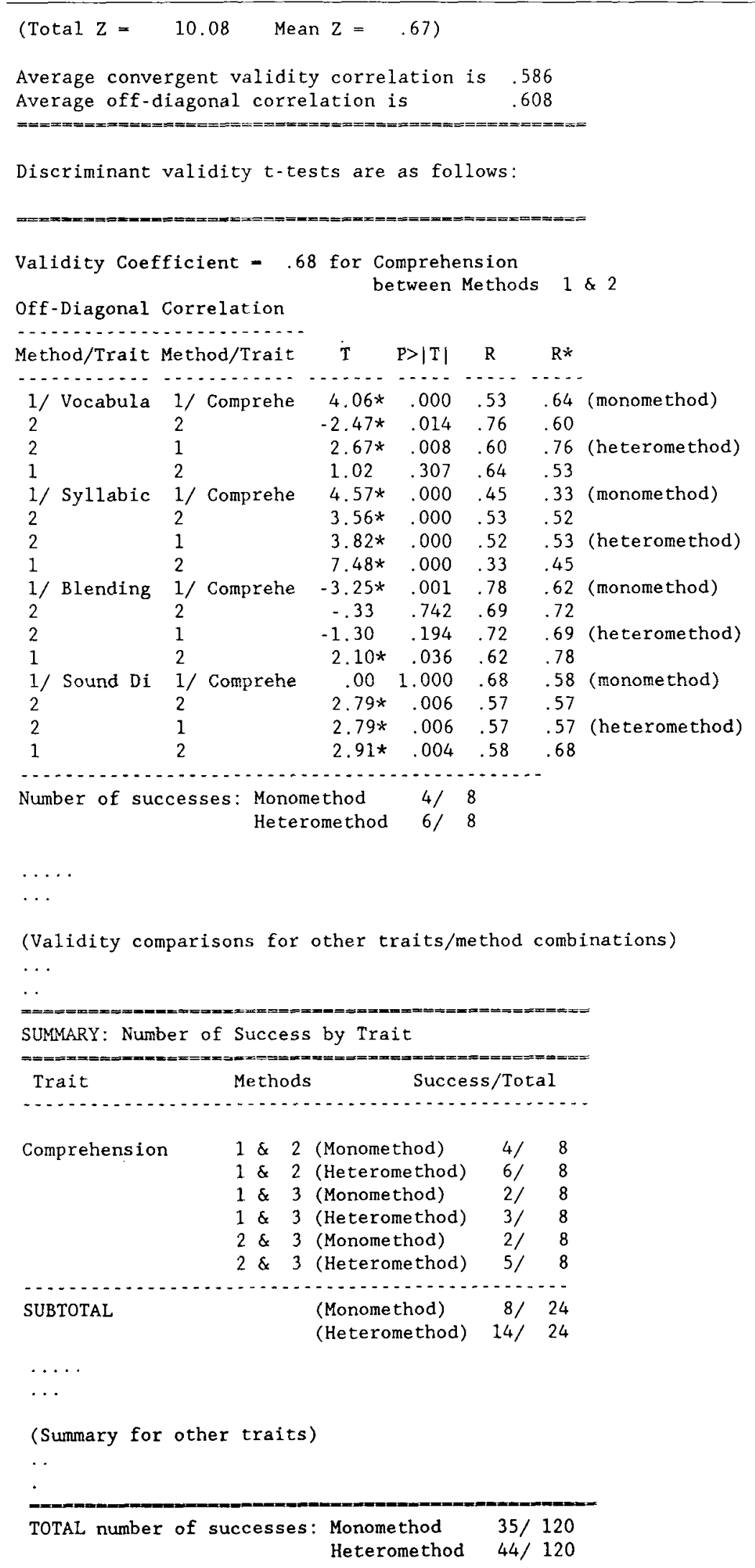


matrix of which is taken from Marsh and Butler (1984). The verbose output style was selected. This style contains four segments: title, input matrix, validity comparisons, and summary table.

Following the title section, a list of the input matrix is provided in an easily readable format, with brackets around validity diagonal correlations and numbers and labels placed on the top and the side.

Validity comparisons are done in an order that helps the user examine discriminant validity systematically. For each validity coefficient (bracketed correlation), the comparisons are presented by trait, and within trait by monomethod and heteromethod. A monomethod comparison is defined as one in which a common method is shared; a heteromethod comparison is one between different methods. For each comparison the program generates relevant correlations, the $t$ statistic recommended by Steiger (1980), and the probability associated with the $t$ statistic. Validity comparisons for each validity coefficient are summarized by the number of successes out of the total number of comparisons, broken down by monomethod and heteromethod. Although comparisons for the other validity coefficients are provided by the program, journal space considerations dictate that only a sample of the output appear here. (All of the output of the program is available upon request.)

The last section of the verbose output summarizes the validity comparison results, showing the number of successes by trait. Within each trait, it further breaks down the results by method and whether it is a monomethod or heteromethod comparison. If the user wants a concise output, the summary output style can be selected to get the summary table alone.

Availability. The compiled MTMM.EXE program and its documentation are available without charge by mailing a MS-DOS (or PC-DOS) formatted diskette and a selfaddressed, postage-paid mailer to the first author.

\section{REFERENCES}

CampBell, D. T., \& FisKe, D. W. (1959). Convergent and discriminant validity by the multitrait-multimethod matrix. Psychological Bulletin, 56, 81-105.

HAYS, R. D. (1986). MTMM.BAS: A program for analyzing multitraitmultimethod matrices. Bulletin of the Society of Psychologists in Addictive Behavior, 5, 23-28.

MARSH, H. W., \& BUTLER, S. (1984). Evaluating reading diagnostic tests: An application of confirmatory factor analysis to multitraitmultimethod data. Applied Psychological Measurement, 8, 307-320.

Steiger, J. H. (1980). Tests for comparing elements of a correlation matrix. Psychological Bulletin, 87, 245-251.

\section{NOTE}

1. The program internally stores data in RAM and does computation. The size of the preallocated data area is $40 \mathrm{~K}$ or 5,050 data elements. To see if a given MTMM matrix is within this range, compute $M=$ $N(N+1) / 2$, where $N=$ (\# of methods) (\# of traits). If $M$ is less than 5,050 , then the MTMM matrix can be analyzed.

(Revision accepted for publication December 21, 1986.) 